\title{
Positive Mass Theorem for Black Holes in Einstein-Maxwell Axion-dilaton Gravity
}

\author{
Marek Rogatko \\ Technical University of Lublin \\ 20-618 Lublin, Nadbystrzycka 40, Poland \\ rogat@tytan.umcs.lublin.pl \\ rogat@akropolis.pol.lublin.pl
}

August 22, 2018

PACS numbers: 04.50.+h, 97.60.L.

\begin{abstract}
We presented the proof of the positive mass theorem for black holes in Einstein-Maxwell axion-dilaton gravity being the low-energy limit of the heterotic string theory. We show that the total mass of a spacetime containg a black hole is greater or equal to the square root of the sum of squares of the adequate dilaton-electric and dilaton-magnetic charges.
\end{abstract}

\section{Introduction}

In general relativity, global quantities as a total energy or total angular momentum of an isolated system do not arise naturaly as in special relativity. There were heavy attempts to prove that the mass of an isolated system is positive. The story began in 1959 when partial results were obtained by Araki [1] and Brill [2]. The first complete proof of the positive energy theorem was deviced by Schoen and Yau [3]. Shortly afterwards, Witten [4] conceived the elegant proof that the ADM mass of an asymptotically flat spacetime containing matter satisfying the dominant energy conditions is non-negative and vanishes in the case of a flat spacetime. His reasoning was based on the analysis of spinors fulfilling a Dirac-like equation on a three-dimensional spacelike hypersurface. Soon after, Parker and Taubes [5] gave a mathematically rigorous proof of the positive energy theorem. They completed and simplified the original arguments presented in [四]. 
Nester's attitude [6] treated the problem of the positive energy mass theorem in a fully covariant way, in order to avoid technical difficulties concerning the three-dimensional truncation of the four-dimensional divergence theorem. In several papers [7] the issue of extending Witten's proof of the positivity of energy at spatial infinity to a proof in the case of null infinity was considered. The authors established the positivity of the Bondi mass. Recently Penrose et al. [8] based on a causal structure and focusing proved the positive mass theorem.

Soon after, the positive energy theorem was established, similar techniques were used to prove several extensions of this result. Gibbons and Hull [9] proved the positive mass theorem for Einstein-Maxwell theory and were able to derive a lower bound for the mass of the spacetime. The positive mass theorem was also generalized to hold in supergravity [10 and in Kaluza-Klein theory [11, 12]. In [13] it was shown that an inclusion of Yang-Mills fields, Yang-Mills-Higgs, dilaton interactions implied that self-gravitating solitons saturated a gravitational version of the Bogomolnyi bound on energy. In paper [14 the proof of the positive mass theorem in the case of the low-energy string theory, i.e, the so-called Einstein-Maxwell axion-dilaton gravity, was presented. A lower bound for the mass of a spacetime was derived in the theory under consideration.

Gibbons et al. [15] extended the positive mass theorem to asymptotically flat manifolds containing black holes. Recently, Herzlich [16] provided the rigorous mathematical proof of the black holes positive mass theorem. In [17] Gibbons extended the Geroch-Wald-Jang-HuiskenIlmanen approach to the positive energy problem and gave a negative lower bound for the mass of asymptotically Anti-de-Sitter spacetimes containing horizons. It was also shown that the method gave a lower bound for the mass of time-symmetric initial data sets for black holes with scalar and vectors. For a review of the positive energy theorem story see, e.g., [18].

In this paper we want to provide some continuity with the work 14 and to some extent generalize it. We shall consider the problem of the black hole positive mass theorem in the so-called Einstein-Maxwell axion-dilaton gravity being the so-called the low-energy limit of the heterotic string theory. Our paper is organized as follows. In Sec.II the basic field equations of the theory under consideration were presented and the non-negativity of the black hole mass was established. Sec.III summarizes our results.

In our paper the metric $g_{\mu \nu}$ has signature $(-+++)$ and $\gamma$ matrices obey the standard condition $\left\{\gamma^{\mu}, \gamma^{\nu}\right\}=2 g^{\mu \nu}, \sigma^{\mu \nu}=\frac{1}{4}\left[\gamma^{\mu}, \gamma^{\nu}\right]$. Greek indices change from 0 to 3, while Latin ones from 1 to 3 . Indices with hats are refered to an orthonormal frame in which $\gamma^{\hat{a}}$ matrices are hermitian and $\gamma^{0}$ antihermitian. 


\section{Black Holes Positive Mass Theorem}

The so-called low-energy limit of superstring theories provides an interesting generalization of the Einstein-Maxwell (EM) theory. A simplified model of this kind in an Einstein-Maxwell axion-dilaton (EMAD) coupled system containing a metric $g_{\mu \nu}, U(1)$ vector fields $A_{\mu}$, a dilaton $\phi$ and three-index antisymmetric tensor field $H_{\alpha \beta \gamma}$. The action has the form 19

$$
I=\int d^{4} x \sqrt{-g}\left[R-2(\nabla \phi)^{2}-\frac{1}{3} H_{\alpha \beta \gamma} H^{\alpha \beta \gamma}-e^{-2 \phi} F_{\alpha \beta} F^{\alpha \beta}\right]+I_{\text {matter }},
$$

where the strength of the gauge fields are descibed by $F_{\mu \nu}=2 \nabla_{[\mu} A_{\nu]}$ and the three index antisymmetric tensor is defined by

$$
H_{\alpha \beta \gamma}=\nabla_{\alpha} B_{\beta \gamma}-A_{\alpha} F_{\beta \gamma}+\text { cyclic }
$$

In four dimensions $H_{\alpha \beta \gamma}$ is equivalent to the Peccei-Quin pseudoscalar and may be written as follows:

$$
H_{\alpha \beta \gamma}=\frac{1}{2} \epsilon_{\alpha \beta \gamma \delta} e^{4 \phi} \nabla^{\delta} a .
$$

As a consequence of the definition (3) the action (1) yields

$$
I=\int d^{4} x \sqrt{-g}\left[R-2(\nabla \phi)^{2}-e^{-2 \phi} F_{\alpha \beta} F^{\alpha \beta}-a F_{\mu \nu} * F_{\mu \nu}\right]+I_{m a t t e r},
$$

where $* F_{\mu \nu}=\frac{1}{2} \epsilon_{\mu \nu \delta \rho} F^{\delta \rho}$. The resulting equations of motion, derived from the variational principle become

$$
\begin{array}{r}
\nabla_{\mu}\left(e^{-2 \phi} F^{\mu \nu}+a * F^{\mu \nu}\right)=\mathcal{J}^{\nu}(\text { matter }), \\
\nabla_{\mu}\left(* F^{\mu \nu}\right)=0, \\
\nabla_{\mu} \nabla^{\mu} \phi-\frac{1}{2} e^{4 \phi} \nabla_{\mu} a \nabla^{\mu} a+\frac{1}{2} e^{-2 \phi} F^{2}=0, \\
\nabla_{\mu} \nabla^{\mu} a+4 \nabla_{\gamma} \phi \nabla^{\gamma} a-e^{-4 \phi} F_{\mu \nu} * F^{\mu \nu}=0, \\
G_{\mu \nu}=T_{\mu \nu}(\text { matter })+T_{\mu \nu}(F, \phi, a),
\end{array}
$$

where $T_{\mu \nu}(F, \phi, a)=\frac{-2 \delta I}{\sqrt{-g} \delta g^{\mu \nu}}$ the energy momentum tensor for $U(1)$ gauge fields, axion and dilaton fields reads

$$
\begin{aligned}
T_{\mu \nu}(F, \phi, a)=e^{2 \phi}\left(4 F_{\mu \rho} F_{\nu}{ }^{\rho}-g_{\mu \nu} F^{2}\right) & -g_{\mu \nu}\left[2(\nabla \phi)^{2}+\frac{1}{2} e^{4 \phi}(\nabla a)^{2}\right] \\
& +\nabla_{\mu} \phi \nabla_{\nu} \phi+e^{4 \phi} \nabla_{\mu} a \nabla_{\nu} a .
\end{aligned}
$$

In what follows we will consider a spacelike hypersurface $\Sigma$, with induced metric $h_{i j}$ imbeded in four-dimensional spacetime $\left(\mathcal{M}, g_{\mu \nu}\right)$. A spacelike hypersurface $\Sigma$ is assumed to be asymptotically flat, i.e., there exists a flat metric $\delta_{i j}$ defined outside a compact set $\mathcal{G}$ such that $\Sigma-\mathcal{G}$ is 
diffeomorphic to the complement of a compact set in $R^{3}, h_{i j}=\delta_{i j}+\mathcal{O}\left(\frac{1}{r}\right), K_{a b}=\mathcal{O}\left(\frac{1}{r^{2}}\right) . K_{a b}$ is an extrinsic curvature of $\Sigma$. The hypersurface element is denoted by $d A^{i}$ while the boundary at spatial infinity by $\partial \Sigma$. One chooses the tetrad so that the zero indices vector is orthogonal to the hypersurface $\Sigma$. The manifold under consideration will contain a black hole and the main task will be to evaluate the mass of the black hole.

In order to do so we introduce the supercovariant derivative acting on a spinor field $\epsilon_{I}$ is given by the formula [20, 14]

$$
\hat{\nabla}_{\mu}^{(4)} \epsilon_{I}=\nabla_{\mu}^{(4)} \epsilon_{I}+\frac{i}{2} e^{2 \phi} \nabla_{\mu}^{(4)} a \epsilon_{I}+\frac{i}{2} e^{-\phi} F_{\alpha \beta} \gamma^{\alpha} \gamma^{\beta} \gamma_{\mu} \alpha_{I K} \epsilon^{K},
$$

where $I, K$ stand for $S O(4)$ indices, $\alpha_{I K}$ is $S O(4)$ matrix [21]. The suprecovariant derivative (11) can be thought as a supersymmetry transformation about non-trivial gravitational, scalar and $U(1)$ gauge backgrounds [10, 14].

Projecting the four-dimensional supercovariant derivative into the hypersurface $\Sigma$ and multiplying the result by $\gamma^{\hat{a}}$ and in the end equating the outcome to zero, one gets the Witten equation given by

$$
\gamma^{\hat{a}} \nabla_{\hat{a}}^{(3)} \epsilon_{I}+\frac{1}{2} K \gamma^{\hat{0}} \epsilon_{I}+\frac{i}{2} e^{2 \phi} \gamma^{\hat{b}} \nabla_{\hat{b}}^{(3)} a \epsilon_{I}-\frac{i}{4} e^{-\phi} \gamma^{\hat{b}}\left(E_{\hat{a}}-2 B_{\hat{a}} \gamma_{\hat{5}}\right) \gamma^{\hat{0}} \gamma^{\hat{a}} \gamma_{\hat{b}} \alpha_{I K} \epsilon^{K}=0 .
$$

where $K=K_{\hat{a}}{ }^{\hat{a}}$ is the triad component of the second fundamental form of the hypersurface $\Sigma$, and $\gamma^{\hat{5}}=\gamma^{\hat{0}} \gamma^{\hat{1}} \gamma^{\hat{2}} \gamma^{\hat{3}},\left(\gamma_{\hat{5}}\right)^{2}=-1$. The adequate components of $F_{\mu \nu}$ have the forms

$$
F_{\hat{b} \hat{0}}=E_{\hat{b}}, \quad F_{\hat{a} \hat{b}}=\epsilon_{\hat{a} \hat{b} \hat{c}} B^{\hat{c}} .
$$

As in the derivation of the positive mass theorem [14], it will be convenient to define the quantity defined as follows:

$$
\delta \lambda_{I}=\gamma^{\alpha} \nabla_{\alpha}^{(4)} \phi \epsilon_{I}+\frac{i}{2} e^{2 \phi} \gamma^{\beta} \nabla_{\beta}^{(4)} a \epsilon_{I}-\frac{i}{8} e^{-\phi} F_{\alpha \beta} \gamma^{\alpha} \gamma^{\beta} \alpha_{I K} \epsilon^{K} .
$$

The motivation for introducing $\delta \lambda_{I}$ is to achieve the desired mass bound for black holes. However, the expression (14) has also the motivation as the supersymmetry transformation laws of the appropriate particles in the associated supergravity model.

Taking into account equations of motion, after lengthy calculations we reached to the indentity

$$
\begin{aligned}
\int_{H} d A^{i}\left(\epsilon^{I}\right)^{\dagger} \hat{\nabla}_{i}^{(4)} \epsilon_{I}+\int_{\Sigma} d \Sigma\left(\hat{\nabla}^{(4) i} \epsilon^{I}\right)^{\dagger} \hat{\nabla}_{i}^{(4)} \epsilon_{I} & + \\
\frac{1}{2} \int_{\Sigma} d \Sigma\left(\epsilon^{I}\right)^{\dagger}\left[T_{\hat{0} \hat{0}}(\text { matter })+T_{\hat{0} \hat{a}}(\text { matter }) \gamma^{\hat{0}} \gamma^{\hat{a}}\right] \epsilon_{I} & -i \int_{\Sigma} d \Sigma e^{-\phi}\left(\epsilon^{I}\right)^{\dagger} \gamma^{\hat{0}}\left(\mathcal{J}_{\hat{0}}^{E}-\gamma^{\hat{5}} \mathcal{J}_{\hat{0}}^{M}\right) \alpha_{I K} \epsilon^{K} \\
+\int_{\Sigma} d \Sigma\left(\delta \lambda^{I}\right)^{\dagger} \delta \lambda_{I}+\int_{\Sigma} d \Sigma\left(\epsilon^{I}\right)^{\dagger} \mathcal{K} \alpha_{I K} \epsilon^{K} & =\int_{S_{\infty}} d A^{i}\left(\epsilon^{I}\right)^{\dagger} \hat{\nabla}_{i}^{(4)} \epsilon_{I}
\end{aligned}
$$


where $\mathcal{J}_{\mu}^{E}$ is the electric current while $\mathcal{J}_{\mu}^{M}$ is the magnetic current. The energy momentum tensor of the matter fields is equal to

$$
T_{\mu \nu}(\text { matter })=T_{\mu \nu}(\text { total })-T_{\mu \nu}(F, \phi, a)
$$

while $\mathcal{K}$ denotes

$$
\begin{aligned}
\mathcal{K} & =e^{-\phi}\left(3 E^{\hat{i}} \nabla_{\hat{i}}^{(3)} \phi \gamma^{\hat{0}}-\epsilon^{\hat{i} \hat{j} \hat{k}} \nabla_{\hat{i}}^{(3)} E_{\hat{j}} \gamma_{\hat{k}} \gamma_{\hat{j}}+2 \epsilon^{\hat{i} \hat{j} \hat{k}} \nabla_{\hat{i}}^{(3)} a B_{\hat{j}} \gamma_{\hat{k}}\right) \\
& +\frac{e^{\phi}}{4}\left(B^{\hat{i}} \nabla_{\hat{i}}^{(3)} a \gamma^{\hat{0}} \gamma^{\hat{5}}-\epsilon_{\hat{i} \hat{j} \hat{k}} B^{\hat{i}} \nabla^{(3) \hat{j}} a \gamma^{\hat{k}}\right) .
\end{aligned}
$$

Now we turn to the question of a spacelike hypersurface $\Sigma$ with an boundary $H$. We shall work in the tetrad which $e_{\hat{0}}$ unit zero-vector is orthogonal to $\Sigma$ and unit one-vector $e_{\hat{1}}$ is orthogonal to $H$. The remainning ones lie in $H$. It happened that one cannot require the condition of vanishing $\epsilon_{I}$ on $H$ to be satisfied. As was discussed in [15], the Witten's equation would imply that the derivative of $\epsilon_{I}$ transversal to $H$ also disappeared. So that one has $\epsilon_{I}=0$ everywhere. Then, we need to restrict the freedom of $\epsilon_{I}$ on $H$. Following [15], one chooses the boundary conditions as follows:

$$
\gamma^{\hat{1}} \gamma^{\hat{0}} \epsilon_{I}-\epsilon_{I}=0
$$

The above condition restricts the freedom of spinors $\epsilon_{I}$ on $H$ by half. It is caused so by the fact that the matrix $\gamma^{\hat{1}} \gamma^{\hat{0}}$ has eigenvalues \pm 1 , with eigenspaces of dimensions equal to two. Taking into account the Witten's equation, $\gamma^{\hat{a}} \hat{\nabla}_{\hat{a}}^{(4)} \epsilon_{I}=0$, admitting a solution satisfying the asymptotic requirements and the boundary conditions (18) we will have a closer look at the surface term on $H$ in equation (15). After some algebraic manipulations, the surface term on $H$ can be written as

$$
\begin{aligned}
& \int_{H} d A^{\hat{c}}\left(\epsilon^{I}\right)^{\dagger} \hat{\nabla}_{\hat{c}}^{(4)} \epsilon_{I}=-\frac{1}{2} \int_{H} d A\left(\epsilon^{I}\right)^{\dagger}\left[\left(K+J-K_{\hat{1} \hat{1}}\right) \gamma^{\hat{1}} \gamma^{\hat{0}} \epsilon_{I}+2 \gamma^{\hat{1}} \gamma^{\hat{A}} \mathcal{D}_{\hat{A}} \epsilon_{I}\right]+ \\
& -\frac{i}{8} \int_{H} d A e^{-\phi}\left(\epsilon^{I}\right)^{\dagger}\left[2 \gamma^{\hat{0}}\left(E_{\hat{1}}-\gamma_{\hat{5}} B_{\hat{1}}\right)\right] \alpha_{I K} \epsilon^{K}-\frac{i}{2} \int_{H} d A e^{2 \phi}\left(\epsilon^{I}\right)^{\dagger} \gamma^{\hat{1}} \gamma^{\hat{A}} \nabla_{\hat{A}}^{(2)} a \epsilon_{I} .
\end{aligned}
$$

where we set $\hat{A}=\hat{2}, \hat{3}$ and $J$ denotes the mean curvature of $H$ in $\Sigma$. By $\mathcal{D}_{\hat{A}} \epsilon_{I}$ we defined 15 the following derivative:

$$
\mathcal{D}_{\hat{A}} \epsilon_{I}=\nabla_{\hat{A}}^{(2)} \epsilon_{I}+\frac{1}{2} K_{\hat{A} \hat{1}} \gamma^{\hat{1}} \gamma^{\hat{0}} \epsilon_{I}
$$

An inspection of equation (18) easily shows that the matrix $\gamma^{\hat{1}} \gamma^{\hat{0}}$ anticommutes with the operator $\mathcal{L}=\gamma^{\hat{1}} \gamma^{\hat{A}} \mathcal{D}_{\hat{A}}$, thus the second term on the right-hand side of equation (19) vanishes if $\epsilon_{I}$ satisfies the boundary conditions. Since then also $\left(\epsilon^{I}\right)^{\dagger}=-\left(\epsilon^{I}\right)^{\dagger} \gamma^{\hat{1}} \gamma^{\hat{0}}$ and $\left(\epsilon^{I}\right)^{\dagger} \mathcal{L} \epsilon_{I}=-\left(\epsilon^{I}\right)^{\dagger} \mathcal{L} \epsilon_{I}$, which implies that $\left(\epsilon^{I}\right)^{\dagger} \mathcal{L} \epsilon_{I}=0$. Applying the same arguments, one finds that matrices $\gamma^{\hat{0}}, \gamma^{\hat{0}} \gamma_{\hat{5}}$ 
anticommute with $\gamma^{\hat{1}} \gamma^{\hat{0}}$ then the third term of expression (19) disappear.

Moreover, if $H$ is a future apparent horizon [22], one has that $K+J-K_{\hat{1} \hat{1}}=0$. If $H$ is a past apparent horizon the same situation takes place [15]. Then, one uses boundary conditions like (18) but with a minus sign.

The last term of the considered equation vanishes if we impose the additional condition for axion fields, namely that there is no axion currents on the horizon surface, i.e., $\nabla_{\hat{A}}^{(2)} a=0$.

Thus we see that the right-hand side of equation (19) vanishes.

Further, we shall assume that the matter energy momentum tensor obeys the following energy condition

$$
T_{\hat{0} \hat{o}}(\text { matter }) \geq\left[T_{\hat{0} j}(\text { matter }) T_{\hat{0}}^{j}(\text { matter })+\left(\mathcal{J}_{\hat{0}}^{E}\right)^{2}+\left(\mathcal{J}_{\hat{0}}^{M}\right)^{2}\right]^{\frac{1}{2}},
$$

and moreover, we impose the additional conditions, as follows:

$$
\delta \lambda_{I}=0, \quad \mathcal{K}=0
$$

The first relation is motivated by the invariance of the entire system under the supersymmetry transformation, while the other inputs relations among the fields appearring in the theory under consideration. The very similar condition was obtained in the energy bounds studies in EinsteinMaxwell-dilaton system [13] and in EMAD system without interior boundaries [14].

Now we would like to remark on the existence of the solutions of equation (12). Following the reasoning presented in [15], instead of dealing with the spinor fields which tend asymptotically to constant values at large distances on $\Sigma$ we take into account a conformal transformation compactify the hypersurface by adding a point at infinity. The metric $\tilde{h}_{i j}$ on a compact manifold $\tilde{\Sigma}$ will be conformally related to the metric on $\Sigma, \tilde{h}_{i j}=\Omega^{4} h_{i j}$. The conformal factor $\Omega$ is required to compactify an asymptotically flat hypersurface $\Sigma$ and it is of the form $\Omega=\frac{1}{r}+\mathcal{O}\left(\frac{1}{r^{2}}\right)$, while $K=\mathcal{O}\left(\frac{1}{r^{2}}\right)$. As we shall take into account the behaviour on the horizon we set $P_{a}=0$ [15]. On the above smooth manifold with the boudary $H$ the spinor fields $\tilde{\epsilon}_{I}=\frac{\epsilon_{I}}{\Omega^{2}}$ obey the relation as follows:

$$
\tilde{\gamma}^{\hat{a}} \tilde{\nabla}_{\hat{a}}^{(3)} \tilde{\epsilon}_{I}+\frac{1}{2} \Omega^{2} K \gamma^{\hat{0}} \tilde{\epsilon}_{I}+\frac{i}{2} \Omega^{2} e^{2 \phi} \gamma^{\hat{b}} \nabla_{\hat{b}}^{(3)} a \tilde{\epsilon}_{I}-\frac{i}{4} \Omega^{2} e^{-\phi} \gamma^{\hat{b}}\left(E_{\hat{a}}-2 B_{\hat{a}} \gamma_{\hat{5}}\right) \gamma^{\hat{0}} \gamma^{\hat{a}} \gamma_{\hat{b}} \alpha_{I K} \tilde{\epsilon}^{K}=0
$$

where $\tilde{\gamma}^{b}=\Omega^{-2} \gamma^{b}$ and $\tilde{\nabla}_{i}$ is the covariant derivative with respect to $\tilde{h}_{a b}$. Suppose that equation (23) has a non-zero solution $\tilde{\epsilon}_{I(1)}$ obeying the boundary conditions (18). Then $\Omega^{2} \tilde{\epsilon}_{I(1)}$ would be a spinor field on $\Sigma$ which satisfies the Witten equation, the boundary conditions on $H$ and which decreases to zero at infinity like $\frac{1}{r^{2}}$. In the light of equation (19) and conditions (21) and (22), it will be a contradiction because of vanishing of the boundary terms. It shows that the conformal Witten's equation with the boundary (18) has no zero modes on $\tilde{\Sigma}$. All these arguments suggest 
that there exist a Green's function to this elliptic boundary value problem [23]. In this way, one can obtain solutions to the Witten's equation on $\Sigma$ fulfilling the boundary conditions (18) and approaching a constant spinor at infinity.

Probably, the existence of a non-zero solution to equation (12) can be rigourously proved by means of an isomorphism between some adapted Sobolev spaces. We hope to return to this question elsewhere.

To complete our considerations concerning equation (15) we shall consider the surface term at infinity. The relationship between the integral of the surface term at infinity and the ADM mass may be demonstrated by considering solutions of the Witten equation. By virtue of the direct generalization of the arguments given in [4], one gets

$$
\int_{S_{\infty}} d A^{i}\left(\epsilon^{I}\right)^{\dagger} \hat{\nabla}_{i}^{(4)} \epsilon_{I}=\frac{1}{2}\left(\epsilon_{\infty}^{I}\right)^{\dagger} M \epsilon_{I \infty}-\frac{i}{8}\left(\epsilon_{\infty}^{I}\right)^{\dagger} \gamma^{\hat{0}}\left(Q_{(F-\phi)}-P_{(F-\phi)}\right) \alpha_{I K} \epsilon_{\infty}^{K},
$$

where $\epsilon_{I \infty}$ is a spinor field which is constant in some chart around infinity. $M$ is the ADM mass defined by $M=\sqrt{P_{A D M} P_{A D M}}$, where $P_{A D M}$ is the ADM four momentum. The dilaton-electric and dilaton-magnetic charges of the black hole are defined respectively as

$$
Q_{(F-\phi)}=\int_{S_{\infty}} d S^{i} e^{-\phi_{\infty}} E_{i}, \quad P_{(F-\phi)}=\int_{S_{\infty}} d S^{i} e^{-\phi_{\infty}} B_{i}
$$

The non-negativity of the left-hand side of equation (15) for any $\epsilon_{I \infty}$, implies that

$$
M \geq \sqrt{Q_{(F-\phi)}^{2}+P_{(F-\phi)}^{2}} .
$$

We have thus proved the positive black hole mass theorem in EMAD gravity.

\section{Conclusions}

In summary, we have studied the positive mass theorem for black holes in EMAD gravity being the low-energy limit of the heterotic string theory. We analysed an asymptotically flat spacelike hypersurface with induced metric $h_{i j}$, containing black holes. We have considered spinors obeying the Dirac type equation on this hypersurface. Using the classical Witten's arguments we show that in four-dimensional manifold satisfying the energy condition (21) and the two other requirements (22) imposed on fields in the theory, the ADM mass of the black hole in the theory under consideration is nonnegative, provided that the square of the total mass of a spacetime containing the black hole is greater or equal to the sum of squares of the total dilaton electric and dilaton magnetic charges of the black hole. 


\section{Acknowledgement}

I would like to thank the unknown referees for very useful comments. 


\section{References}

[1] Araki H, 1959 Ann.Phys.7 456.

[2] Brill D, 1959 Ann.Phys.7 466.

[3] Schoen R and Yau S-T 1979 Commun.Math.Phys. 6545.

[4] Witten E 1981 Commun.Math.Phys. 80381.

[5] Parker T and Taubes C.H 1982 Commun.Math.Phys. 84223.

[6] Nester J 1981 Phys.Lett.A 83241.

[7] Nester J and Israel W 1981 Phys.Lett.A 85 259, Ludvigsen M and Vickers J 1981 J.Phys.A 14 L389, Ludvigsen M and Vickers J 1981 J.Phys.A 15 L67, Horowitz G and Tod K.P 1982 Commun.Math.Phys. 85 429, Horowitz G and Perry M 1982 Phys.Rev.Lett. 48371.

[8] Penrose R, Sorkin R and Woolgar E 1993 "A Positive Mass Theorem Based on the Focusing and Retardetion of Null Geodesics", Preprint gr-qc 931015.

[9] Gibbons G.W and Hull C.M 1982 Phys.Lett.B 109190.

[10] Deser S 1983 Phys.Rev.D 27 2805, Deser S 1984 Phys.Rev.D 30 1392, Hull C.M 1983 Commun.Math.Phys. 90 545, Gibbons G.W, Hull C.M and Warner N.P 1983 Nucl.Phys.B 218173.

[11] Moreshi O.M and Sparling G.A.J 1984 Commun.Math.Phys 95 113, Moreshi O.M and Sparling G.A.J 1986 J.Math.Phys. 272402.

[12] Lee J and Sorkin R.D 1988 Commun.Mat.Phys. 116353.

[13] Gibbons G.W, Kastor D, London L.A.J, Townsend P.K and Trashen J 1994 Nucl.Phys.B 416850 .

[14] Rogatko M 1995 Class.Quantum Grav. 123115.

[15] Gibbons G.W, Hawking S.W, Horowitz G.T and Perry M.J 1983 Commun.Math.Phys. 88 295. 
[16] Herzlich M 1998 J.Geom.Phys. 2697.

[17] Gibbons G.W 1998 "Some Comments on Gravitational Entropy and the Inverse Mean Curvature Flow", Preprint hep-th 9809167.

[18] Choquet-Bruhat Y 1984 Relativity, Groups and Topology II, ed.B.S.DeWitt and R.Stora (North Holland).

[19] Shapere A, Trivedi S and Wilczek F 1991 Mod.Phys.Lett.A 292677.

[20] Kallosh R, Linde A, Ortin T, Peet A and van Proyen A 1992 Phys.Rev.D 465278.

[21] Cremmer E and Scherk J 1978 Phys.Lett.B 7461.

[22] Hawking S.W and Ellis G.F.R 1973 The Large Scale Structure of Space-Time (Cambridge University Press, Cambridge).

[23] Hörmander L 1988 The Analysis of Linear Partial Differential Operators, III (Springer, Berlin). 